\title{
Aus der Geschichte Europas auf dem Weg zu einer europäischen Verfassung
}

\author{
Markus Vette
}

Am 1. Mai 2004 werden zehn Staaten neu in die Europäische Gemeinschaft (EU) aufgenommen.

Die Europäische Gemeinschaft, ursprünglich aus sechs Partnern gebildet, wurde zwar bereits mehrfach erweitert und umfasste im Jahr 200315 Mitgliedsstaaten, aber eine so umfangreiche Herausforderung wie die Erweiterung 2004 und die Reform der Institutionen stand ihr sicher noch nicht bevor. Denn mit der kommenden Erweiterung besteht die Chance, dass alle europäischen Völker ihre Angelegenheiten erstmals dauerhaft und institutionalisiert friedlich miteinander regeln können. Um kein Missverständnis aufkommen zu lassen: Der Friede selbst gilt nicht als Grundwert in der Gesellschaft der Bundesrepublik Deutschland. Grundwerte sind Freiheit, Solidarität und Gerechtigkeit. Aus dem Umgang mit diesen Grundwerten, aus deren Ausgestaltung erwächst der gesellschaftliche Frieden. ${ }^{1}$ Gleichwohl berühren und bewegen der Friede und seine Erhaltung die Völker enorm, zwei Weltkriege im 20. Jahrhundert haben sich tief in das Gedächtnis der europäischen Völker eingeprägt. Diese Geschichte und Entwicklung muss eine Wertegemeinschaft, wie die Europäische Union eine ist, immer reflektieren. Das Recht in der Europäischen Union resultiert ja aus den gemeinsamen Werten und wirkt auf diese zurück. Es regelt das gesellschaftliche Zusammenleben in Wirtschaft, Sozialem, in Kultusangelegenheiten, von einzelnen Bürgern und kollektiven Akteuren. Europäisches Recht und europäisches Management sind keine abgehobenen theoretisierenden Versuche, sondern strukturieren zunehmend das gesellschaftliche Leben. Vor dem Blick auf die zukünftigen Entwicklungen ist ein Blick in die Geschichte geboten.

\section{Aus der Geschichte Europas}

Zum Verständnis der Geschichte Europas muss man sich die Mühe machen, die Geschichte der Völker Europas differenziert zu betrachten. Als Leitfaden dafuir kann das Buch „Die Begründung Europas“ von Ferdinand Seibt ${ }^{2}$ dienen. Seibt gibt einen „Zwischenbericht über die letzten 1000 Jahre“. Darin verortet ${ }^{3}$ er Europa in einem Dreieck, an dessen Spitzen Gibraltar, der Polarkreis und die eurasischen Steppen liegen.

Bis etwa zum Jahr 1000 betrachtet der Autor vor allem den Mittelmeerraum, der im Norden natuirlich begrenzt wird durch die Alpen. Die Besatzung und Kultur des römischen Reiches in den geografischen Ausmaßen würde stark mit einer modernen Weinkarte übereinstimmen, bemerkt Seibt. ${ }^{4}$ Bedeutendste politische Gestalt war wohl um 800 Karl der Große, mit dem ein Dualismus von
Kaiser und Papst entstand. Handelswege führten entlang der Flüsse, die Alpenpässe hatten Monopolfunktion, oft mit Klöstern zur Beherbergung der Reisenden. Um die Jahrtausendwende (962) wurde das Heilige Römische Reich geschaffen und zunächst von den Ottonen mit Zentrum in Magdeburg 5 regiert, dann von den Saliern (Speyer), den Staufern (Rothenburg), den Luxemburgern (Prag und Nürnberg) und schließlich bis 1804 von den Habsburgern (Wien).

Alle Wege der europäischen Priester führten nach Rom, so auch Martin Luther, die der europäischen Adligen als Kreuzfahrer (mit eigenem Rechtsstatus) nach Jerusalem und die der armen sämtlich gläubigen Menschen vornehmlich als vom Gesetz geschützte Pilger auf dem Jakobsweg in das spanische Santiago. 6 Damit sind die drei wichtigsten Wallfahrtswege genannt. Kirchliche Erneuerungsbewegungen wurden verketzert oder führten zu neuen Orden, die etwa als Zisterzienser beginnend im 13. Jahrhundert die Mark Brandenburg rodeten, meliorierten und kultivierten.

Die Hanse als Städtebund ging zu Grunde, oberitalienische Städte vermochten sich dagegen auch politisch zu behaupten.

Die Kirche war vor 1000 eine Klosterkirche, erst später eine weltliche Predigerkirche. Seit 1054 war sie zwischen dem westlichen Rom und dem östlichen Konstantinopel, dem zweiten Rom, gespalten, seit Luther zusätzlich zwischen dem nördlichen lutherischen Wittenberg und dem katholischen Rom, die anglikanische Kirche nahm zeitnah eine separate Entwicklung. Der Sieg des Papstes im Investiturstreit von 1077 in Canossa war ein gefährlicher Sieg, denn er sollte sich langfristig als Niederlage erweisen. Im Heiligen Römischen Reich wählten seit 1356 die sieben Kurfürsten in Frankfurt/Main den Kaiser, in Aachen wurde er inthronisiert, bis 1530 wurde der Kaiser zuvor vom Papst in Rom gesalbt.

Mit Beginn des 16. Jahrhundert wird gewöhnlich der Begriff einer Zeitenwende verbunden. Die großen Entdeckungen, die Ausbreitung des Buchdruckes, die Reformation und Kopernikus werden genannt. Spanien und Portugal konnten zunächst am meisten von den neuen Ländern profitieren. Im 17. Jahrhundert wurde der konfessionell benannte Konflikt 30 Jahre lang ausgetragen, seine Folgen reichen bis weit in die zweite Hälfte dieses Jahrhunderts.

England köpfte 1648 seinen König, lange bevor die Franzosen den ihren köpften. Die Französische Revolution 1789-93 formte die Nation und diskreditierte zugleich den Demokratie-Begriff durch den Revolutionsterror, sie läutete das 19. Jahrhundert ein, das Jahrhundert des Bürgertums, der Industrialisierung, die Entstehung der sozialen Frage und beinahe abschließend die deutsche 
Reichsgründung 1871. In Deutschland ging der politische Kampf wiederholt um eine Verfassung, die dann doch mit Blut und Eisen zustande kam.

Im 20. Jahrhundert gingen von Deutschland zwei Weltkriege aus. Zum Ende des 20. Jahrhunderts hat aber gerade Deutschland die Chance, den deutschen Einigungsprozess mit dem europäischen zu verbinden. So sollen hier einige Entwicklungslinien zum Geschichtsverständnis der beiden großen Nachbarn Deutschlands, Frankreich und Polen, vorgestellt werden.

\section{Aus der Geschichte Frankreichs}

Das Reich Karls des Großen wurde bekanntlich unter seinen Enkeln in drei Teile gegliederte, das westliche spätere Frankreich, das östliche Reich, aus welchem später Deutschland entstand und in das dazwischen liegende nach Lothar benannte Reich, später Lothringen, welches sich von der Nordsee bis an das Mittelmeer erstreckte. Nach Seibt wurden wichtige kulturelle Entwicklungen gerade in diesem mittleren, verbindenden Reich vermittelt.

Im 14. Jahrhundert stand das Papsttum im Exil in Avignon unter französischer Vorherrschaft. In Frankreich konnte sich nach langen Kämpfen mit England schließlich eine Zentralgewalt herausbilden, Paris wurde das Zentrum des Landes. Die Absicht König Franz' I., 1519 zum Kaiser des Heiligen Römischen Reichs Deutscher Nation gewählt zu werden, war erfolglos. Das änderte nichts daran, dass Franz I. in den kriegerischen Auseinandersetzungen der Reformationszeit mit den Päpsten und Kaiser Karl V. immer wieder eine aktive Rolle spielte, man musste mit ihm rechnen.

Obwohl nach dem Augsburger Religionsfrieden von 1555 bis 1618 die formal betrachtet längste Friedensperiode in Europa folgte, wirkte natuirlich die Reformation Luthers und Calvins erheblich in der Gesellschaft, man erinnere sich an die Bartholomäusnacht in Paris von 1572 und das Edikt von Nantes von 1598, dessen Aufhebung 1685 zur Emigration der Hugenotten, speziell nach Brandenburg, fuihrte. Durch die enormen Verluste und Belastungen der deutschen Fürstentümer ${ }^{7}$ während des Dreißigjährigen Krieges 1618 bis 1648 gelangte nicht zuletzt wieder der Nachbar Frankreich in eine politisch offensive Position: Das katholische Frankreich und das protestantische Schweden fungierten als Schutzmächte des Westfälischen Friedens von Münster und Osnabrïck. Diese Rolle hielt weder Ludwig XIV. von Frankreich davon ab, 1681 die deutsche Reichsstadt Straßburg zu besetzen, noch Schweden, die Mark Brandenburg und Pommern heimzusuchen. Der Übergriff der Schweden wurde bekanntlich durch den Großen Kurfürsten von Brandenburg mit der Schlacht bei Fehrbellin 1675 abgewehrt.

Mit der Französischen Revolution 1789 bis 1793 gab es eine neue Agenda in der europäischen Politik. Auf deren Verbindungen mit der amerikanischen Unabhängigkeitserklärung von 1776 bzw. der amerikanischen Verfassung von 1787 soll hier nicht eingegangen werden.

Das lange 19. Jahrhundert war besonders in Frankreich ein Jahrhundert wechselnder Konstitutionen, in Deutschland war es bis Bismarck nur das Jahrhundert des Wun- sches nach einer Konstitution. ${ }^{8}$ In Frankreich zählt man gegenwärtig die 5. Republik, deren Entstehung und Entwicklung zwei Kaiserreiche und zahlreiche Verfassungen zugrunde liegen, was einen Exkurs in die französische Verfassungsentwicklung nahe legt. ${ }^{9}$

Mit dem deutsch-französischen Krieg 1870/71 wurde Elsass/Lothringen deutsch besetzt und Lothringen geteilt, es begann das Denken in den Kategorien des Erbfeindes auf der Ebene der Nation. Im besetzten Gebiet wurde man zum Deutschen, es sei denn, man wählte bzw. optierte für den Weg ins Innere Frankreichs und wurde so zum Optanten. Im Ersten Weltkrieg folgten die Schlachten von Sedan und Verdun, wovon angeblich ,im Westen nichts Neues"10 zu berichten gewesen sei. An der deutsch-französischen Grenze wurden in den zwanziger Jahren durch einen französischen Kriegsminister, André Maginot, 58 Befestigungsanlagen mit 400 Bunkern und 1,5 Millionen Tonnen Beton errichtet, 5 Milliarden Franc wurden verbaut. 30000 Mann Besatzung sollten diese Grenze verteidigen. ${ }^{11}$ Man mag sich selbst einen Eindruck vom Ausmaß der Grenzbefestigungen an der französischen Maginot-Linie im Elsass und in Lothringen sowie vom deutschen West-Wall verschaffen, wenn man etwa die Festung Bitche (deutsch Bitsch) unmittelbar hinter der deutsch-französischen Grenze mit den gewaltigen unterirdischen Sälen und Verbindungen besichtigt. ${ }^{12}$ Die Festung in Bitche prägt nach heute das Stadtbild.

Im Zweiten Weltkrieg wurde Frankreich weit durch deutsche Truppen besetzt. General de Gaulle organisierte den militärischen Widerstand und gehörte schließlich zu den alliierten Siegern. Umso wichtiger war, dass gerade de Gaulle und Adenauer nach dem Zweiten Weltkrieg die deutsch-französische Aussöhnung praktizierten, die Montanunion schufen, dass Frankreichs Außenminister Robert Schuman und sein Mitarbeiter Jean Monnet für die Entwicklung der europäischen Gemeinschaft eintraten. Straßburg wurde ein Zentrum und Kristallisationspunkt der europäischen Einigung. So findet man etwa heute noch im Straßburger Münster, dem seinerzeit höchsten Bauwerk der Christenheit, historische FensterMalereien sowohl von Heiligen als auch von Kaisern, die zur Legitimation der jeweiligen Macht und zur Belehrung des Volkes dienten, sowie in der Apsis ein Marienbild mit der Europa-Flagge im Hintergrund. Ein großer Zeitrahmen wird so vermittelt.

\section{Aus der Geschichte Polens}

Nach dem Exkurs zum westlichen Nachbarn nun zum östlichen. Für den Umgang mit den polnischen Nachbarn sollten Kenntnisse der polnischen Geschichte vorhanden sein.

Im Jahre 1000 errichtete Kaiser Otto III. in Gnesen ein Erzbistum, welches die polnische Hauptstadt war. 1024 wurde Boleslaw zum König gekrönt. Bald darauf wurde fuir ein halbes Jahrtausend Krakau zur polnischen Hauptstadt. Die Heiratspolitik verband zunächst polnische und deutsche Herrscherhäuser. Zwischen 1320 und 1795 bestand das polnische Königtum. Im 13. und 14. Jahrhun- 
dert kam es zu Städtegründungen „nach Magdeburger Recht“ bzw. nach deutschem Recht, womit eine gewisse Selbstverwaltung klassifiziert wird.

Seit dem 13. Jahrhundert lebte und kämpfte im Land der Pruzzen der Deutsche Orden, der u. a. die mächtige Marienburg in Elbing errichtete. Sein Einfluss ging nach der Niederlage bei Tannenberg 131410 schrittweise zurück. 1525 wandelte der Hochmeister des Deutschen Ordens, Albrecht von Ansbach-Brandenburg ${ }^{14}$, den Ordensstaat in ein weltliches Herzogtum um, nachdem er in den Jahren zuvor den Reformator Martin Luther kennen gelernt hatte.

Im 16. Jahrhundert blühte Krakau mit der von den Jagellionen errichteten Universität auf. 1543 erschien das Buch „De revolutionibus orbitum...“ des Thorner Kanonikers Kopernikus. Seit dem 16. Jahrhundert wurde der polnische König durch den Adel gewählt, dessen erforderliche Einstimmigkeit zu verhindern eine fuir interessierte Kräfte lösbare Aufgabe war, wie dies selbst der Preußenkönig Friedrich II. zwei Jahrhunderte später in seinem politischen Testament von 1752 freimuitig niederlegte. Im Dreißigjährigen Krieg wurde Polen - zumindest im Vergleich zum benachbarten Brandenburg - verhältnismäßig verschont, denn es blieb neutral. Später musste es sich der Expansion Schwedens als Schutzmacht des Westfälischen Friedens erwehren und verband sich mit Russland. 1683 konnte Jan III. Sobielski in der Schlacht am Kahlenberg das bedrohte Wien (und damit das christliche Abendland, wie vielleicht Luther formuliert hätte) von den türkischen Truppen befreien. 1697 wurde August II. von Sachsen polnischer König, wie in jenen Jahren der Kurfuirst von Hannover die englische und der Kurfürst von Brandenburg die preußische Krone erwarb. August II. blieb König bis 1704. Ihm folgte 1704 bis 1710 Stanislaw Leszczynski. August II. war wieder 1710 bis 1733 König, Leszczynski wieder 1733. Von 1733 bis 1763 war August III. König, ihm folgte mit Stanislaw August Poniatowski der letzte polnische König von 1764 bis zur dritten polnischen Teilung 1795. Warschau wurde zur Hauptstadt ausgebaut. Man baute in Warschau ähnlich wie zuvor im barocken Dresden.

1740 überfiel Friedrich II. Schlesien, das er sich in drei Kriegen endgültig aneignen sollte. Mit einem eroberten Münzprägestempel überschwemmte er den polnischen Staat mit gefälschten Münzen. Am 3. Mai 1791 verabschiedeten die Polen die erste Verfassung im Kontext der Französischen Revolution, noch vor den Verfassungen der Franzosen. Nur die amerikanische Verfassungsurkunde von 1787 war zu diesem Zeitpunkt bereits verabschiedet.

Preußen, Österreich und Russland vollzogen von 1772 bis 1795 mit den drei Teilungen Polens die Auflösung des polnischen Staates. Dies geschah im Vorfeld und im Kontext der französischen bürgerliche Revolution. Das Wahlkönigtum Polens wurde an die Wettiner gekoppelt, die Verfassung ein halbes Jahr vor der französischen verabschiedet. Der Saal im Warschauer Königsschloss, in dem die Verabschiedung stattfand, ist heute zu besichtigen und zeigt eine Faksimile der Verfassungsurkunde.

Der Inhalt der Verfassungsurkunde, insbesondere die Regelungen zur Religion, zum Staatsaufbau und zur
Gewaltenteilung werden nun kurz vorgestellt. Dem modernen Leser mögen die Formulierungen in dieser Verfassungsurkunde unspektakulär erscheinen und geläufig sein, seinerzeit war dies anders: Bereits in der Präambel wird eine doppelte Legitimation des Königs verankert, sowohl „von Gottes Gnaden“ als auch „durch die Stände“. Es sei daran erinnert, dass im „Heiligen Römischen Reich deutscher Nation“ Reichstagsabschlüsse durch „Kaiser und Reich“, letzteres vertreten durch die drei Stände der Kurfürsten, Fürsten und Städte, zusätzlich differenziert nach zwei verschiedenen Konfessionen, zustande kamen. Auch ist dem historisch informierten Leser die Weigerung des Preußenkönigs Friedrich Wilhelm IV. von 1849 in Erinnerung, die Wahl zum deutschen Kaiser durch die Frankfurter Nationalversammlung anzunehmen. Er wollte weiter ,von Gottes Gnaden“ und der Zustimmung der Fürsten legitimiert bleiben.

Obwohl in der polnischen Verfassung als herrschende Religion die katholische bezeichnet wird, bekennt man sich zur Religionsfreiheit. Es folgen Verfassungsartikel zu den Ständen, also den Edelleuten und dem Landadel, den Städten und Städtern sowie den Bauern und Landleuten. Letzteren wird die „völligste Freiheit“ verkündet.

Weitere Regelungen betreffen die Regierung, den Reichstag, der aus einer Landbotenstube und einer Senatorenstube besteht, den König als vollziehende Gewalt und die Rechtssprechung. Der Landbotenstube obliegt die Gesetzgebung, in der Senatorenstube wird der König von Bischöfen, Woiwoden ${ }^{15}$, Ministern u. a. beraten. Der polnische Thron soll auf immer ein Familienwahlthron sein, der Inhaber auf Gott, die Nation und die Verfassung seinen Eid leisten. Für die richterliche Gewalt wird ein neuer Codex in Aussicht gestellt. Die Erziehung der Kinder des Königs als erste Kinder der Nation erfolgt zu Religion, Liebe, Tugend und zum Vaterland. Schließlich sollen die Bischöfe am 8. Mai (dem kirchlichen Gedenktag des Hl. Stanislaus) den Verfassungstag zelebrieren, der christlichen Erziehung der Nation dienend, eine Kirche soll gebaut und geweiht werden.

Eine Verfassungswidersetzung oder ein Verfassungsbruch sind gerichtlich zu ahnden.

Diese Verfassungsurkunde vom 03.05.1791 war die erste demokratische Verfassungsurkunde in der Verfassungsgeschichte Europas. Mit einem Gottesdienst bekräftigt wurde sie in der St. Johannes-Kathedrale in Warschau. Auch auf Gemälden ist dies dokumentiert. Wirksam wurde die Verfassung nicht, denn Polen wurde 1772, 1793 und endguiltig 1795 geteilt. Russland erhielt den Osten (bis nahe Warschau), Österreich den Süden (mit Krakau), und Preußen den Westen (mit Gnesen), um die drei genannten historischen polnischen Hauptstädte beispielhaft zuzuordnen. Bis 1918 gab es keinen polnischen Staat als Völkerrechtssubjekt. Wohl gerade deshalb ist aus dieser Zeit ein reiches kulturelles Erbe des polnischen Volkes zu verzeichnen, das an seiner nationalen Identität festhielt und sich auf anderen als der politischen Ebene artikulierte. ${ }^{16}$

Woodrow Wilson forderte 1918 die Gründung eines unabhängigen polnischen Staates mit einem freien Zugang zum Meer. Der in Magdeburg internierte Pilsudski kehrte nach Polen zuruick, wurde Oberbefehlshaber und Staats- 
präsident. Nach dem Ersten Weltkrieg gab es unter der Maxime von Woodrow Wilson, den Völkern ihr Selbstbestimmungsrecht einzuräumen. Nationalistische Auseinandersetzungen in Gebieten, wo sowohl deutsche und polnische Menschen lebten, waren die Folge. In die Minderheit geratene Volksgruppen wurden, wie 1871 im Elsass und in Lothringen, vielfach zu Optanten, die ihre Heimat verließen. 1919 wurde eine „Kleine Verfassung“ verabschiedet. 1921 fanden in Oberschlesien Volksabstimmungen über die künftige Zugehörigkeit entweder zu Deutschland oder zu Polen statt. 60 Prozent stimmten für Deutschland, Oberschlesien wurde daraufhin geteilt, Kattowitz wurde polnisch. Von 1919 bis 1939 gab es ein selbständiges Polen.

Durch einen Staatsstreich von Pilsudski wurde 1926 ein autoritäres System eingefuihrt. 1935 beschränkte eine neue polnische Verfassung das Parlament auf eine reine Beratungsfunktion. Im September 1939 wurde Polen erneut geteilt. Dem Nichtangriffspakt von Hitler und Stalin am 23.08.1939 folgte der deutsche Überfall auf Polen am 01.09., die Sowjetarmee besetzte am 17.09.1939 die Sowjetrussland zugewiesenen Gebiete. Es folgten deutsche Besatzung, Willkuir, Deportation, Vernichtung in Konzentrations- und Vernichtungslagern in bislang unvorstellbarem Ausmaß. Die Erschießung von 4000 polnischen Offizieren durch sowjetische Geheimdienste in Katyn wurde erst Ende der 1980er Jahre öffentlich. 1943 fielen beim Aufstand im Warschauer Ghetto 60000 Menschen, beim Warschauer Aufstand 1944 verloren 200000 polnische Menschen ihr Leben.

Im ostpreußischen Rastenburg verbaute man wie an der Grenze zum „Erbfeind“ Frankreich Unmengen von Beton fuir Hitlers Kriegsquartier. Im Zweiten Weltkrieg wurden schwerste Verbrechen an polnischen und jüdischen Menschen begangen, das Verhältnis der Nachbarn schien irreparabel zerstört. Nach Kriegsende wurden Menschen zwangsweise umgesiedelt: Aus dem früheren polnischen Osten, der an die Sowjetunion fiel, mussten Menschen in das nunmehrige westliche Polen an Neiße und Oder umsiedeln. Der Grenzverlauf zwischen dem deutschen und dem polnischen Staat wurde an Oder und Neiße bewusst kurz gewählt und festgelegt. Die deutschen Bewohner mussten ihrerseits von dem nun polnischen Gebiet nach Westen in das kleiner gewordene Deutschland wechseln. Polen und die DDR waren bald fest im sowjetischen Machtbereich verankert, die Bundesrepublik gehörte zur freien westlichen Welt. Sie ging den Weg der West-Integration.

In Polen wird die Nachkriegszeit bis zum Ende der Volksrepublik Polen gern in fünf zeitlich zu unterscheidende Etappen ${ }^{17}$ untergliedert:

1. Die Zeit zwischen Juli 1944 und Dezember 1948 wird als die Periode zwischen der Befreiung und der Implementierung des Einparteienstaates bezeichnet. an die Macht kam schlussendlich die zwangsweise Vereinigte Polnische Arbeiterpartei (PVAP), nachdem polnische Kommunisten und Linke verschiedener Prägung noch wenige Jahre zuvor ihre eigenen negativen Erfahrungen mit der Kaderpolitik des Generalissimus Stalin machen mussten.

2. Die Jahre 1948 bis 1956 werden als die Jahre des pol- nischen Stalinismus bezeichnet. 1947 wurde eine vorläufige, am 22.07.1952 eine Verfassung der Volksrepublik verabschiedet. Auf dem Papier standen bürgerliche Freiheiten, Wahlen, eine parlamentarisch verantwortliche Regierung und mehr, in der Wirklichkeit lag die Macht beim Politbüro und der Nomenklatura. Tausende Todesurteile und Zehntausende in politischen Lagern sind in diesen acht Jahren zu beklagen.

Als architektonisches Relikt aus dieser Zeit grüßt den Besucher in Warschau immer noch der Kulturpalast, eine kleine Stadt für sich im Herzen der polnischen Hauptstadt.

Wie das ostdeutsche Stalinstadt, heute Eisenhuittenstadt, verfügt Polen über eine ähnlich neu angelegte Stadt, Nowa Huta nahe Krakau, der demonstrativ 20 Jahre lang der (landesüblich katholische) Kirchbau verweigert wurde.

Mit der Anerkennung der Oder-Neiße-Grenze durch die benachbarte DDR wurde 1950 eine wichtige außenpolitische Entscheidung fuir Polen getroffen. 1953 bis 1956 war der fuihrende polnische Bischof ${ }^{18}$, Kardinal Wyszynski, in Haft, da er jede politische Einflussnahme der herrschenden KP auf die Kirche zurïckwies. Wyschinski als Primas der katholischen Kirche in Polen sollte später seinem Krakauer Kollegen Wojtyla prophezeien, er würde die katholische Kirche in das dritte Jahrtausend führen.

3. 1956 kam der 1948 abgesetzte Gomulka wieder an die Parteispitze, was ein besonderer Vorgang ist. Er hatte zuvor den Posener Aufstand gefuihrt. Ihm gelang es, mit der katholischen Kirche, den freien also nicht kollektivierten Bauern und einer Reihe weiterer politischer Maßnahmen einen gewissen Kompromiss zwischen den maßgeblichen politischen Kräften im Lande zu erreichen. Dieser Kompromiss sollte bis 1980 tragen, wobei von 1970 bis 1980 Edward Gierek Parteischef war. 1970 kamen bei Arbeiteraufständen in Gdansk, Gdingen und Sopot Arbeiter ums Leben. 1970 wurde zwischen der Bundesrepublik Deutschland und Polen der Warschauer Vertrag abgeschlossen, dessen Ratifizierung im Deutschen Bundestag eine harte innenpolitische Debatte mit sich brachte. 1972 gab es den Kniefall Willi Brandts in Warschau. Des Parteichefs Gierek Erfolgsrezept sollten eigentlich internationaler Handel und Weltwirtschaft werden, tatsächlich war das Land in kurzer Zeit hoffnungslos verschuldet sowie den Schwankungen der Weltwirtschaft ausgesetzt und diesen in keiner Weise gewachsen. 1975/76 machten Verfassungsänderungen Polen auch formal per Verfassungstext zum Staat unter der Vorherrschaft der Sowjetunion.

4. Als vierte Etappe ist die Periode der Gewerkschaft Solidarnosc von August 1980 bis Dezember $1981 \mathrm{zu}$ bezeichnen. Im Oktober 1978 wurde der Krakauer Kardinal Wojtyla zum Papst gewählt. Am 31. August 1980 trotzten die Danziger Werftarbeiter mit dem Elektriker Lech Walesa der Regierung politische Rechte ab, Solidarnosc erreichte mit zehn Mio. Mitgliedern praktisch jede polnische Familie, jedes dritte KP-Mitglied war auch Mitglied der Solidarnosc-Gewerkschaft. Nach den polnischen Unruhen von 1980, als der Arbeiterführer Walesa in Danzig der Regierung 
eine Vereinbarung abringen konnte, wurden in der DDR wieder antipolnische Ressentiments bemüht und vorsorglich private Kontakte nach Polen unterbunden.

5. Die Militärdiktatur General Jaruzelskis ${ }^{19}$ dauerte vom 13.12.1981 bis zum 22.07.1983. Der wirtschaftliche Niedergang wurde beschleunigt, am 08.10.1982 wurde die Gewerkschaft Solidarnosc endguiltig verboten. Die polnische Volksrepublik existierte noch bis zum Runden Tisch und den ersten freien Wahlen 1989, die zu einem überwältigenden Sieg der Gewerkschaftskräfte der Solidarnosc führten. Mazowiecki wurde erster nichtkommunistischer Regierungschef.

1989 fiel die Mauer in Berlin, wenige Tage später, am 12.11.1989, fand in Kreisau auf dem Gut der Moltkes ${ }^{20}$ ein Gottesdienst der Versöhnung statt, an dem Polens erster nichtkommunistischer Regierungschef Mazowiecki und Bundeskanzler Kohl teilnahmen. Letzterer war am Tag des Mauerfalls, dem 09.11.1989, in Warschau zu Gast.

Zehn Jahre später, 1999, ist Polen Mitglied der NATO, 15 Jahre später, im Jahre 2004, wird Polen EU-Mitglied sein. - Seit 1991 gibt es den deutsch-polnischen Vertrag über gute Nachbarschaft und freundschaftliche Zusammenarbeit. Am 07.12.1992 bekam die Republik Polen eine neue „kleine Verfassung“, am 17.10.1997 trat die neue Verfassung in Kraft. Der polnische Präsident wird für fünf Jahre gewählt, das Parlament besteht aus zwei Kammern: Im Sejm sitzen 460 Abgeordnete, im Senat gibt es 100 Sitze. Diese beiden Kammern werden alle vier Jahre gewählt. Das Referendum zum EU-Beitritt im Mai 2003 brachte die Zustimmung der polnischen Bevölkerung.

Wenn man heute in Warschau die Altstadt mit dem Schloss und dem Alten Markt besucht, trifft man viele Polen, vor allem Schulklassen, die nicht nur den Ort der ersten Verfassungsverabschiedung, das Warschauer Schloss, sondern auch den Ort der feierlichen Bestätigung dieser Verfassung, die Kirche ${ }^{21}$,Johannes der Täufer" $^{22}$ in der St. Johannes-Straße ${ }^{23}$ unweit des Schlosses besuchen. Auf dem Weg zum historischen Alten Marktplatz Warschaus ist die Kirche nicht zu übersehen. Man begeht in Polen also nicht unbedingt den 1. Mai als Arbeiterfeiertag, allenfalls vielleicht im Verständnis der Heiligen Josefs als Patron der Arbeiter in der katholischen Kirche: Unmittelbar nach dem Ende der kommunistischen Herrschaft knuipfte man dagegen an die Verabschiedung der ersten geschriebenen europäischen Verfassung vom 03. Mai 1791 an. Der 3. Mai ist heute ein polnischer Nationalfeiertag.

\section{Auf dem Weg zu einer europäischen Verfassung}

Natürlich besitzen nicht nur das französische und polnische, sondern auch die anderen europäischen Völker, die zur Union gehören bzw. gehören werden, ihre Geschichte. Diese dienen ebenfalls als Rechtserkenntnisquellen, ihre Darstellung kann aber hier nicht erfolgen. Wenn es darum geht, nach der im westlichen Teil Europas über 1000 Jahre gewachsenen Verbindung von der Bre- tagne über die Rheinschiene nach Italien nun die Osterweiterung der Europäischen Union zu vollziehen, die im übrigen Deutschland und speziell Brandenburg in die Mitte Europas (wieder zurück) positioniert, sollte man sich Kenntnisse über die europäischen Partner und speziell die unmittelbaren großen Nachbarn aneignen. Im Saarland, Rheinland-Pfalz, Luxemburg, Elsass, und Lothringen versteht man sich als eine Region. ${ }^{24}$ Der deutschfranzösische Kulturkanal arte ist etabliert. Die deutschfranzösische Aussöhnung im Südwesten der Bundesrepublik seit 1950 mag als Erkenntnisquelle dienen, um die deutsch-polnische Aussöhnung vor allem im Nordosten der Bundesrepublik ab 1990 erfolgreich in Angriff zu nehmen.

Gerade die Beschäftigung mit der Geschichte der großen Nachbarn Deutschlands belegt: Das friedliche Zusammenleben der europäischen Völker ist in einer Wertegemeinschaft möglich. Statt Kriegsbefestigungen kann man andere Bauwerke errichten. Statt die Heimat als Optanten verlassen zu müssen, kann man für das friedliche Zusammenleben der Menschen optieren und dieses unter Berücksichtigung der geschichtlichen Entwicklung der Völker regeln bzw. managen.

Der europäische Konvent mit dem Ziel, eine europäische Verfassung zu erarbeiten, hat eine äußerst anspruchsvolle Aufgabe. Es geht dabei nicht nur um das Europarecht im engeren Sinne, sondern um das Zusammenleben der Menschen und Völker im umfassenden Sinne, wozu Wissen, Kenntnisse und nicht zuletzt der Respekt vor den Partnern gehören.

\section{Ausgewählte weiterführende Literatur}

Davies, Norman: Heart of Europe, Oxford, new edition, 2001. Escher, Felix; Vietig, Jürgen: Deutsche und Polen, nicolai, 2002. (ORB-TV-Produktion).

Lebe, Reinhard: Ein Königreich als Mitgift, dtv 30792, 2000.

Masing, J.: Die polnische Verfassung von 1791; in: Juristenzeitung Jg. 57, Nr. 9, S.428

Regionalverwaltung Lothringen (Hrsg.): 2000 Jahre Lothringische Geschichte, 2000.

Reinalter, Helmut (Hrsg.): Die polnische Verfassung vom 3. Mai 1791 vor dem Hintergrund der europäischen Aufklärung, Frankfurt am Main 1997.

Seibt, Ferdinand: Die Begründung Europas, S. Fischer, 2002. Streinz, Rudolf: Europarecht, C.F.Müller, 1999.

Vette, Markus: Eine europäische Verfassung nach Vorbild des deutschen Grundgesetzes? - ...; Lehrunterlage Europarecht TFH Wildau, 2000.

Vette, Markus: Der Persönlichkeit nach bekannt..., BoD 2002.

\section{Anmerkungen}

1 „Gerechtigkeit schafft Frieden“ hieß eine These des ökumenischen Prozesses in den 80er Jahren.

2 Die Darstellung ist weniger auf Daten, Herrscher und Schlachten fixiert, sondern bietet vielmehr umfangreiche wirtschaftliche, soziale und kulturelle Bezüge, sie bildet den Leser in einem umfassenden Sinne.

3 Als Zeit werden 1000 Jahre, als Raum bzw. Ort dieses geografische Dreieck genannt. 
4 Seibt, Begrüindung Europas, S. 56.

5 Im Jahr 2002 gab es eine große Ausstellung zu Otto I. in Magdeburg.

6 Santiago de Compostella liegt im Nordwesten Spaniens.

7 Zu diesem Zeitpunkt waren die Elemente des Staates (noch) nicht gegeben, weshalb der Begriff des Staates etwa für Brandenburg vor Mitte des 17. Jahrhunderts nicht verwendet wird.

8 Die Existenz der Verfassungen des süddeutschen Raumes aus der Zeit von 1820 darf nicht darüber hinwegtäuschen, dass die fuihrenden Staaten des deutschen Bundes, Österreich und Preußen, der Verfassungsforderung der Bundesakte nicht, spät oder restriktiv nachkamen.

9 s. Vette, Markus: Lehrunterlage Europarecht TFH Wildau, 2000.

10 Remarque, E. M. und andere Autoren ; Hinweise in Vette, M. : Der Persönlichkeit nach bekannt.

112000 Jahre Lothringische Geschichte, S. 95.

12 Vette, M.: Der Persönlichkeit nach bekannt...

13 Eine der ersten Schlachten des Ersten Weltkrieges ist mit dem Namen Tannenberg verbunden. Die Polen nennen diese Schlacht „Schlacht bei Grunwald“. Hitler fuihrte am Tannenberg-Denkmal Feierlichkeiten durch.

14 Nicht zu verwechseln mit dem Erzbischof Albrecht von Brandenburg in Mainz, Kurfürst und Kardinal, an den sich Luther 1517 mit seinen Thesen wandte.

15 Verwaltungschef der untergeordneten Behörde auf regionaler Ebene.

16 Man sollte nicht übersehen, dass im 19. Jahrhundert auch in Deutschland eine reichhaltige kulturelle und wissenschaftliche Blüte zu verzeichnen war, erst im letzten Drittel des 19. Jahrhunderts wurde der deutsche Nationalstaat politisch durchgesetzt.

17 Anlehnung an Norman Davies: Heart of Europe, The Past in Poland's Present, 2001, das in Warschau mehrfach als das beste polnische Geschichtsbuch bezeichnet wurde.

18 Es wird auch die Bezeichnung Primas verwendet.

19 Erst auf einer Festveranstaltung der ARD zum 30. Jahrestag des Warschauer Korrespondentenstudios wurde Anfang Februar 2003 festgestellt, dass General Jaruzelski in jedem Falle als polnischer Patriot gelten kann.

20 Der Freundeskreis der Hitlergegner um James Graf von Moltke wird als Kreisauer Kreis bezeichnet.

21 Es handelt sich um die Warschauer Bischofskirche, die Kathedrale, auf alten Stadtplänen als „Große Kirche“ bezeichnet.

22 Polnischer Kirchenname: Bazylika Archekatedralno sw. Jana Chrzciciela.

23 Polnischer Straßenname: Swetojanska.

24 Die Region heißt Saarlorlux.

Hinweis: Redaktionsschluss dieses Beitrags März 2003.

\section{Autor}

\section{Dr. Dr. Markus Vette}

Technische Fachhochschule Wildau

Fachbereich Wirtschaft, Verwaltung und Recht

Telefon +493375 508-972

E-Mail: mvette@wvr.tfh-wildau.de 